\title{
Fast and efficient: Statistical learning predicts online reading times in a second language
}

\author{
Luca Onnis ${ }^{\mathrm{a}, *}$, Hongoak Yun ${ }^{\mathrm{b}}$ \\ ${ }^{a}$ University of Genoa, Italy \\ ${ }^{b}$ Jeju National University, South Korea
}

\begin{abstract}
Two long-standing and interrelated questions have been central to language and cognition. The first question is whether shared or separate mechanisms subserve language learning versus language processing. The second question is whether shared or separate mechanisms underlie processing in a first versus second language. Using an individual differences paradigm, we sought evidence for common mechanisms by probing implicit statistical learning (SL) skills and online reading in a second language. We found that individuals with better statistical learning skills more efficiently incorporate word-level statistical regularities while reading in their second language, making them more efficient L2 readers. In addition, sensitivity to forward and backward lexical predictability reduced reading times. Thus, sensitivity to statistical sequential structure may be a common mechanism of implicit learning as well as processing of language. Keywords: Individual differences, Statistical learning, Language processing, Expectation-based models, Artificial language learning, Second Language Reading
\end{abstract}

\section{Introduction}

Human languages are learnt and processed in real time. The speech signal is the ultimate fleeting experience, as it dissipates as soon as it is produced or heard. And while printed text is stationary, proficient readers process words

*Corresponding author: luca.onnis@unige.it

Preprint submitted to Studies in Second Language Acquisition

February 19, 2020 
sequentially and fluidly, with relatively few gazes spent looking back to reread previous words, and many words skipped altogether. The average silent reading rate for adults in English was recently estimated at 238 words per minute (wpm) for non-fiction and $260 \mathrm{wpm}$ for fiction (Brysbaert, 2019). This inherent fleeting nature of language processing suggests that the brain recruits mechanisms efficiently tuned to processing sequential information. One way that such mechanisms may work is to adapt to the probabilistic nature of language, such that words are processed faster when they are more predictable in a given context. Accordingly, increasing evidence has mounted over the last few decades that people continuously use context to predict how an utterance might continue and then compare these predictions with what they subsequently encounter. Alternatively, a comprehender may combine linguistic information that is activated as a result of processing the input, with a representation of the preceding input, resulting in faster integration when the preceding input is retrodictively more probable given the current word. Prediction and integration/retrodiction in native speakers have been shown to occur at all linguistic levels from semantics to form (Pickering \& Gambi, 2018; Willems et al., 2016). At the simplest level, more frequent and more predictable linguistic units are easier to process than infrequent or less predictable units, and these frequency and predictability effects occur in language comprehension as well as production.

How do speakers and comprehenders develop sensitivity to contextual predictability about the input reflecting different degrees of likelihood of co-occurrence? This knowledge must be based on learned and accumulated long-term linguistic experience, and as such it is legitimate to ask whether similar mechanisms operate for language processing and implicit sequential statistical learning (SL). SL mechanisms involve the ability to unconsciously track and extract patterns of regularities across sensory modalities, and to abstract over these patterns (for reviews see Gómez \& Gerken 2000; Perruchet \& Desaulty 2008). Recent studies have started to relate individual variance in SL tasks involving artificial languages with individual variance in several linguistic tasks involving actual linguistic stimuli. The objective of this approach is to show that some measure 
of statistical learning ability, as assessed in tasks requiring implicitly learning relations among probabilistic sequences, is correlated with performance on one or more tasks involving language. As a plausible corollary, SL could increase language processing by assisting the cognitive system in capitalizing on predictable input during reading or listening.

Here we build on this approach in seeking evidence for a relation between statistical learning and sentence reading in adults. Arguably, statistical language learning does not stop in the early years of childhood. Studies with older children have linked poor implicit statistical skills with language and/or reading difficulties (Evans et al. 2009) and adult native speakers are even sensitive to the particular statistical distribution of sentence structures within an experimental session, and adapt their processing preference accordingly (Fine et al. 2013: Fraundorf \& Jaeger, 2016). Thus, sensitivity to the statistical structure of language is likely to support not only children learning a language, but also proficient adaptive day-to-day communication. Indeed, direct predictive relations between statistical learning scores and online sentence processing and other linguistic tasks exist now both for children and adults (Yim \& Windsor, 2010). In addition, neurophysiological data suggest that similar neural mechanisms serve both syntactic processing of language and statistical learning of sequential patterns Abla et al. 2008, Christiansen et al. 2012. An individual's ability to track implicit sequential regularities in linguistic and nonlinguistic stimuli seems to be independent of factors other than language performance, such as age, nonverbal IQ, and memory (Kaufman et al., 2010).

Our first goal in this paper is thus to seek evidence that mechanisms that underlie statistical learning are also responsible for the sensitivity to statistical processing of natural language. Our specific hypothesis is that individual differences in SL abilities should correlate with sensitivity to conditional word probabilities, which involves processing words sequentially as a sentence unfolds. The probability of a word is conditioned on many aspects of its context, including neighboring words, syntactic and lexical structure, semantic expectations, and discourse factors. In this paper we focused on a particular domain of prob- 
abilistic linguistic knowledge: the role of local probabilistic relations between words, measured as forward and backward conditional probabilities. Conditional (or transitional) probability operationalize the dependency of a word on its immediate context, and thus reflect an estimate of how likely a word occurs, given its right or left neighbouring word. Here word conditional probabilities were estimated by means of a computational linguistic model that assigned occurrence probabilities based on a large representative corpus of English to all words that might come before or next at each point in a sentence. Building on previous findings, a more efficient reader should adapt her reading times to the words' log-transformed occurrence probability, such that more probable words are read more quickly (Goodkind \& Bicknell, 2018, Levy, 2008, Smith \& Levy, 2013, Staub, 2015). Hence, we take the extent to which higher log-transformed transitional probability (as opposed to base word frequency) predicts shorter reading time as indicative of a reader's efficiency.

Our second and related goal is to seek evidence for the generality of distributional mechanisms in learning and processing beyond one's first language (L1), extending it to a second language (L2). Although sensitivity to predictable context plays an important role in L1 processing, its role in an L2 is less clear: Is L2 processing also based on probabilistic prediction and integration mechanisms? Second languages are often learned in different circumstances to first languages, and another long-standing debate at the heart of research into multilingualism is whether L1 and L2 are subserved by different mechanisms or are qualitatively similar. One line of evidence suggests that L2 learners fail to anticipate information during processing, or at least, do so to a lesser extent than native speakers do (Dussias et al., 2013, Grüter \& Rohde, 2013, Grüter et al., 2012, Hopp, 2013. Lew-Williams \& Fernald, 2010; Martin et al., 2013). For example, in a study by Martin et al. (2013), L2 comprehenders, unlike L1 comprehenders, failed to show the classic brain signature (an N400 amplitude increase) for unexpected articles. As the N400 has been viewed as a brain signature indicating sensitivity to word predictability, L2 comprehenders would seem to be unable to predict upcoming words during sentence comprehension to the same extent as L1 comprehenders. 
In another study, adult English learners of Spanish did not use the determiner as a predictive cue in a visual world eye-tracking paradigm, even though they knew the rules of gender concord in Spanish and the correct gender of the nouns that were used (Grüter et al. 2012). Similarly, Mitsugi \& Macwhinney (2016) found that L2 Japanese speakers did not use case-marking (dative/accusative) to predict in a visual world study. However, other studies have observed equal predictive abilities between native-like and L2 learners (Dussias et al., 2013 Hopp, 2013), and the inconsistent evidence leaves the debate unsettled.

More in general, the word-by-word processes that make a second language reader fluent in real time are still relatively understudied. Empirical studies have focused on predictive factors of single word decoding, or higher-order literacy skills such as reading comprehension in young L2 readers (e.g., Erdos et al. 2011)). Working memory (WM) has figured centrally in many theories of second language (L2) proficiency development and processing (Ito et al., 2018), with recent meta-analysis suggesting that it has a robust, positive relationship with L2 outcomes (Linck et al. 2014). Consequently, little is still known about whether statistical learning specifically is an individual ability predicting variation in second language reading. A study by Frost et al. (2013) found that SL predicted single word decoding abilities in a second language. Arciuli \& Simpson (2012) found that more effective SL was related to higher L1 reading ability in children and adults alike. Here again, though, reading was measured as the participants' ability to read aloud individually presented orthographic strings in their first language. Other studies found relations between SL and skills that are critical to the development of literacy, such as oral language, vocabulary knowledge, and phonological processing (Spencer et al., 2015). While foundational to establishing links between the ability to extract statistical regularities in artificial stimuli and reading in natural languages, these findings are limited to learning novel words in isolation, and therefore cannot speak to the mechanisms underlying incremental reading of sentences in real time. In addition, among these studies mentioned above only the Frost study focused on L2. One L2 study that focused on prediction in sentence reading (Leal 
et al. 2017) found that adult second language learners could utilize contextual information to anticipate upcoming grammatical material, a result that dovetails with prediction-based models of language. In particular, they found that English-speaking learners of Spanish were sensitive to syntactic markers of topicalization, which appear early in the sentence and are substantially more frequent in Spanish than in English. However, as the authors point out their data cannot tease apart what specific mechanisms underlie the contextual sensitivities found in their L2ers. Thus, in summary, the literature reviewed so far calls for further clarifications and empirical evidence for the nexus between probabilistic accounts of language processing and statistical learning abilities in second language users.

\section{The Current Study}

Our study measured - in the same Korean L1 / English L2 speakers - their SL abilities in implictly extracting statistical regularities in a novel artificial language learning task, followed by their sensitivity to English-language statistics in an online sentence reading task. In the SL task, participants were first exposed to a stream of spoken monosyllabic pseudowords, organised in probabilistic sequences. Next, they chose which of two word pairs forms an intuitively natural sequence in the artificial language. The extent to which their choices deviated from randomness was taken as a measure for the amount of novel statistical learning that had occurred.

In the subsequent reading task, we measured how strongly word-reading times are predicted by word probability. Do participants rely on predictable context (as measured by forward and backward conditional probabilities, see below) to reduce reading times? If so, this would indicate that they are better at incorporating relevant language-specific statistics in their second language. In addition, if participants with higher SL scores (as measured in the artificial language learning task) showed a stronger relation between word probability and reading time, this would suggest that similar expectation-based mechanisms 
underlie both learning artificial languages and processing natural ones.

\subsection{Participants}

We recruited 58 adult native speakers of Korean (45 women; age $\mathrm{M}=22.6$, $\mathrm{SD}=2.7)$ at four universities in Seoul. We asked participants to report their official scores of English as a second language exams (e.g., TOEFL, TOEIC). The average TOEFL score was 114 (iBT version on a scale of 0 to 120 points) equivalent to 650 in the old PBT version (on a scale of 310 to 677 ), and the average TOEIC score was 950 (on a scale from 10 to 990 points). All participants had experienced living in English-speaking countries for a sustained period. In particular, 40 out of 58 participants had attended English international schools in their preschool, elementary, or secondary school years in countries like the US, UK, Singapore, France, China, Japan, and Guatemala. The average length of stay in these environments was 6 years and 9 months (8 participants had lived abroad more than 10 years). The remaining participants had taken intensive English courses in the US, Canada, and Australia while attending university for 1-3 years. All participants were tested individually in a quiet room at their own university in Seoul and were paid 10,000 Korean Won for their participation.

\subsection{Reading task}

\subsubsection{Materials and Procedure}

Sentences came from the 361-sentence UCL corpus (Frank et al. 2013) explicitly created to evaluate language models on word-reading times. These sentences were drawn from original English narratives. Each participant was randomly assigned to one of ten groups, each containing 36 unique test sentences in English from the University College London UCL corpus, and five practice sentences. Test sentences were presented in random order. The words were displayed one at a time, progressing across the screen in their natural position with successive presses of the spacebar. Approximately half of the sentences were followed by a yes-no question regarding the content of what was just read in order to maintain the attention of the participants. 
Our aim was to investigate to what extent sensitivity to lexical predictability affected word durations. Predictability was measured as two independent predictors, FTP (probability of the currently read word given the preceding word) and BTP (probability of the preceding word given the currently read word. Notice that this is a different measure of BTP used in other psycholinguistic work, namely BTP as the probability of the target word given the following word (Jurafsky et al., 2001; McDonald \& Shillcock, 2003b a; Moers et al., 2017).

Estimating occurrence probabilities of words in context requires a probabilistic language model that implements knowledge about the language's statistics. Possibly the simplest language model is the bigram model, which assumes that a word's probability depends only on its overall frequency and on the immediately preceding word. Hence, word probabilities under such a model equal forward and backward transitional probabilities, which have been found to predict reading times McDonald \& Shillcock (2003b); Onnis et al. (2020); Onnis \& Huettig (2020). More sophisticated models can capture language statistics more accurately, resulting in more accurate reading time predictions (e.g. Frank|2013). For the current study, however, we limited ourselves to a bigram model because the statistical learning task is defined in terms of transitional probabilities. Processing difficulty is proportional to the amount of information conveyed. Surprisal is an information-theoretic measure that estimates how unexpected a given event is. Conceptually, improbable, i.e. 'surprising' events carry more information than expected ones, so that surprisal is inversely related to probability, through a logarithmic function, $\mathrm{S}=(-\log$ (Transition Probability)). Word frequency and forward transitional probability were computed from word and bigram counts in the written-text part of British National Corpus. For example, the forward surprisal of go out is:

Forward $\mathrm{S}($ go out $)=-\log \mathrm{P}($ out $\mid$ go $)=-\log (($ Freq $($ go out $) /$ Freq $($ go $))$

Backward surprisal was calculated based on the backward transitional probability, namely the likelihood of a context preceding a word. Again using the example above, the relevant comparison of backward Surprisal was: 
Backward $\mathrm{S}($ go out $)=-\log \mathrm{P}($ go $\mid$ out $)=-\log (($ Freq $($ go out $) /$ Freq (out $))$

It is worth noting that predictive (forward-going) and retrodictive (backwardgoing) relations among elements of the input are not necessarily identical; for example, whereas the does not strongly predict dog (because many words can follow the), dog more strongly retrodicts the. Thus, while previous research has predominantly considered forward transitional probabilities (and related measures such as forward surprisal) to model reading times, we believe backward probabilities are an independent and complementary source of probabilistic information in reducing lexical ambiguity (for greater detail see Onnis et al. 2020. Onnis \& Huettig 2020).

\subsection{Artificial Language Learning task}

\subsubsection{Materials}

The artificial language lexicon was composed of eight monosyllabic spoken

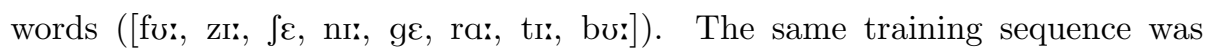
presented to all participants. It was generated by arranging words in a seamless sequence according to the rules of a stochastic Markovian grammar chain. The process started by choosing one of the eight possible words ([fur $]$ ), and then generating the next word according to two probabilistic constraints: whenever the forward probability between any two adjacent words was low (Fwd-TP $\approx$ $.33)$, the backward probability was high $(\mathrm{Bwd}-\mathrm{TP}=1)$, and vice versa. A sample of this sequence is " [... fo: $\int \varepsilon$ zI: ra fo: nI: zI: bv: fo: ge zI: ra fo: nI: zI: bu: fo: ge zI: tr: fo: $\int \varepsilon$ zI: ...]". Frequencies of individual words, bigrams (twoword sequences), and their associated transition probabilities are summarized in Table 1 ,

The speech sequence was realized using the speech synthesizer MBROLA (Dutoit et al., 1996), and concatenating the eight words to form a 3.5 minute speech stream of 711 words, with $80 \mathrm{~ms}$ for consonants and $260 \mathrm{~ms}$ for vowels. Because we were interested in the perception of grouping boundaries as driven by statistical biases alone, MBROLA did not use any prosodic or temporal cues 
Table 1: Summary of statistical relations among adjacent words in the grammar used in the statistical learning task. Fwd-TP and Bwd-TP indicate forward and backward transitional probabilities among words in each bigram. Freq1 and Freq2 indicate frequency of occurrence of first and second word in each bigram, while Freq Bigram indicates how often each bigram occurred during training.

\begin{tabular}{|c|c|c|c|c|c|c|}
\hline Bigram & Type & Fwd-TP & Bwd-TP & Freq1 & Freq2 & Freq Bigram \\
\hline$\left[f_{\mho:} \int \varepsilon\right]$ & LoHi & 0.36 & 1.00 & 133 & 48 & 48 \\
\hline$\left[f_{v:} \mathrm{nI}\right]$ & $\mathrm{LoHi}$ & 0.32 & 1.00 & 133 & 42 & 42 \\
\hline$[f v: g \varepsilon]$ & $\mathrm{LoHi}$ & 0.32 & 1.00 & 133 & 42 & 42 \\
\hline [zI: ra] & $\mathrm{LoHi}$ & 0.35 & 1.00 & 132 & 46 & 46 \\
\hline$[\mathrm{ZII} \quad \mathrm{rI}:]$ & $\mathrm{LoHi}$ & 0.31 & 1.00 & 132 & 41 & 41 \\
\hline [ZI: bv:] & $\mathrm{LoHi}$ & 0.34 & 1.00 & 132 & 45 & 45 \\
\hline$\left[\int \varepsilon \mathrm{ZI}\right]$ & HiLo & 1.00 & 0.36 & 48 & 132 & 48 \\
\hline [nI: ZI: $]$ & HiLo & 1.00 & 0.32 & 42 & 132 & 42 \\
\hline$\left[\begin{array}{ll}g \varepsilon & \mathrm{ZII}\end{array}\right]$ & HiLo & 1.00 & 0.32 & 42 & 132 & 42 \\
\hline$[\mathrm{ra} \mathrm{fv:}]$ & HiLo & 1.00 & 0.35 & 46 & 133 & 46 \\
\hline [tI: for $]$ & HiLo & 1.00 & 0.31 & 41 & 133 & 41 \\
\hline [bv: fv: $]$ & HiLo & 1.00 & 0.34 & 45 & 133 & 45 \\
\hline
\end{tabular}


to grouping boundaries. In addition, the sequence faded in and out for $5 \mathrm{~s}$, giving the impression of an unbounded stream. One Korean and one English adult native speaker not participating in the experiment ensured that all syllable sequences were clearly perceivable in Korean and English respectively.

At test, two types of syllable bigrams were pitted one against the other in a forced-choice task, corresponding to a pattern of high Fwd-TP and low BwdTP (dubbed 'HiLo' bigrams) versus the opposite 'LoHi' bigrams. For example, the LoHi bigram "[for: $\left.\int \varepsilon\right]$ " was presented against the HiLo bigram "[ $\int \varepsilon$ zI $]$ ". Six test-pair trials were presented in random order, while the order within a pair was counterbalanced by repeating each test pair twice, for a total of 12 test trials. Note that HiLo and LoHi bigrams were composed of the same words and had been presented with an equal frequency at training. Hence, the only statistics useful to systematically choose one type over the other would have to be a preference for the patterns of transition probabilities giving rise to the bigrams. Thus, the rationale behind the task was to test whether participants systematically use a pattern of transitional probabilities in parsing the speech stream.

\subsubsection{Procedure}

All stimuli were delivered via a desktop PC. Participants sat comfortably in front of the screen in a quiet room and wore headphones. They were asked to listen to a novel language without further specification. Words would be played in rapid sequence for about 3 minutes. Their task was to listen attentively to this language. After this task they would also be asked questions about what words they had heard. At test they were presented with the forced-choice task between pairs of LoHi and HiLo bigrams. For each pair they were asked to choose, by pressing one of two buttons on the keyboard, what words go together well in the language they had heard. 


\section{Results}

\subsection{Artificial Language Learning task}

The 2AFC test task presented an equal number of items with high forward transitional probabilities and low backward transitional probabilities (HiLo test items) and items with low forward transitional probabilities and high backward transitional probabilities (LoHi test items). Both the HiLo and LoHi items had occurred equally often during training, so the difference between them was the directionality of statistical coherence. Experimental work using artificial input has demonstrated that learners are sensitive to informative relations in both directions (Jones \& Pashler, 2007, Pelucchi et al., 2009, Perruchet \& Desaulty, 2008). We thus defined a participant's statistical learning bias as the proportion of LoHi choices over the 12 test trials presented. The strength of the learning bias, computed as (learning bias -0.5$)^{2}$, quantifies bias extremeness towards either LoHi or HiLo preference. Both the mean statistical learning bias and learning bias strength were above chance level (respectively $M=0.571, t(56)$ $=2.784, p<0.01)$, and $M=0.044, t(56)=6.49, p<0.001)$, suggesting that as a group participants had grouping preferences based on transition probabilities. We noted a bimodal distribution in the distribution of learning bias scores across participants, with the two modes located at 0.4 and 0.6, i.e. on each side of the chance level value of 0.5 . This bimodal distribution suggests that the absolute strength of learning bias may be a better measure reflecting sensitivity to SL.

\subsection{Online Reading task}

\subsubsection{Research questions}

We investigated two questions: first, are English L2 readers sensitive to the statistics of English, namely basic word probability but also forward and backward transition probabilities when reading sentences? This finding would support the view that L2 reading is akin to L1 reading, as it functions in an expectation-based manner and relies on mechanisms of statistical inference and prediction. 
The second research question was, is L2 readers' sensitivity to natural language statistics related to their statistical learning abilities, as assessed independently by the statistical learning task? In particular, we expected that individuals who exhibited a stronger SL preference would be those who showed overall lower reading times. This finding would suggest that similar mechanisms for picking up statistics during learning are used during the word-to-word processing of language in real time.

Extreme Reading Times on a word (below $80 \mathrm{~ms}$ or above $3000 \mathrm{~ms}$ ) were excluded from analyses. Furthermore, we did not include data on sentence-initial and sentence-final words, words followed by a comma, and clitics. This left a total number of 27,000 data points for analysis. To investigate how readers' sensitivity to language statistics is related to their online reading, the collected data were analyzed by linear mixed-effects regression. The fitting engine used for the model was function lmer from R (R Core Team, 2019) package lme4 (Bates et al. 2015). The dependent variable was log-transformed reading time $(\log R T)$ for each word. We considered the following predictors. Itemspecific predictors were: word position in the sentence (WORDNUM), number of letters of target word (NRCHAR), log-transformed word frequency of current work (LOGFREQ), the forward surprisal (negative log-transformed forward transitional word probability, FTP), and backward surprisal (negative log-transformed backward transitional word probability, BTP).

Properties of the previous word (PREVNRCHAR and LOGPREVFREQ) were also included in the mixed model to take into account potential spillover in the reading times. Subject-specific predictors (fixed effects) were: strength of statistical learning bias (SLSTRENGTH) and self-reported second language reading proficiency (L2READ). As trial-specific predictor, log-transformed RT on the previous word (LOGPREVRT) was included to factor out the autocorrelation between consecutive key presses (Baayen \& Milin, 2010). All predictors were scaled and centred and tested for collinearity. Given that word properties are often highly correlated, we calculated variance inflation factor (VIF) and excluded highly correlated predictor variables from the set through 
a stepwise procedure using package fmsb (Nakazawa, 2007), keeping all variable with a VIF $<5$. As a result, log-transformed word frequency of current work (LOGFREQ) and previous word (LOGPREVFREQ), were left out. In addition, the model included by-subject and by-item (i.e., word token) random intercepts and by-subject random slopes of all predictor variables except for the subject-specific one (SL and L2READ).

Using R package buildmer (Voeten, 2019), random factors were included over all terms as long as the model converged; these terms were entered in the order of their contribution to the log-likelihood, such that when the model eventually failed to converge, the most information-rich random intercepts and slopes had been included. From this maximal model, terms were excluded in backward stepwise order based on the change in BIC.

The results of the analyses are shown in Table 2 and in Figures 1 and 2 . As expected, longer words (NRCHAR) had longer latencies, a general psycholinguistic finding. Words that appear later in the sentence (WORDNUM) took less to read, suggesting that participants capitalized on the increasing amount of contextual information to speed up reading. This indicates a consistent finding that comprehenders analyze each word as they encounter it and integrate it with prior context in a highly incremental fashion (Marslen-Wilson, 1973). As more words in a sentence are being read, the content is less ambiguous and reading latencies diminish.

In addition, word reading times were larger when the previous word was read more slowly (LOGPREVRT), reflecting auto-correlative processes typically found in non-independent time series (Baayen \& Milin, 2010). Crucially for our initial hypotheses, there was an effect of FTP such that more predictable words were read faster, reflecting predictive processes as those found in L1 reading. In addition, we found an effect of BTP such as that a more predictable previous word lead to shorter latencies on the current word. This likely reflects processes of postdiction or integration, which were found recently in L1 reading (Onnis et al. 2020; Onnis \& Huettig, 2020). Thus, it appears that L2 reading exhibits probabilistic-based processes that optimize the time it takes to read sentences 
based on lexical conditional probabilities. Furthermore, there was an effect of SLstrength, such that individuals with a stronger SL preference in the artificial grammar task reduced their reading times. In addition, the effect of L2READ indicates that individuals who rated themselves as more proficient readers read words faster.

In addition, a number of interactions were significant (see Table 22. The effect of word position in the sentence affected reading times more for individuals with a stronger SL preference. In particular, these individuals appear to use contextual information more as it accrues over the sentence, in order to reduce reading times. This effect is to be expected given that each sentence stimulus was independent and came from unrelated sources, and thus the beginning of each sentence was by definition less constrained and less predictable than its end. FTP and BTP interacted such that the effect of forward surprisal was larger when backward surprisal was smaller, and viceversa. One interpretation of this finding is that readers use forward and backward contextual information complementarily, relying on the most informative cue at any given point in a sentence. For example, in reading 'big sister', 'sister' may be read fast due to its higher BTP compared to lower FTP. At the same time, in reading 'doctoral student', 'student' may be read faster due to its higher FTP (given that 'student' is relatively predictable after 'doctoral').

Finally, the effect of FTP was modulated by L2 reading abilities, such that it was stronger for less proficient readers. Conversely, the effect of BTP was stronger for more proficient L2 readers. This pattern of results leaves open the possibility that L2 readers become sensitive to forward and backward statistics differentially as a function of experience. Forward probabilities may be easier to internalize than backward probabilities, so they may be more directly available even to less proficient readers. 
Table 2: ANOVA table reporting type 3 effects of the linear regressions predicting log Reading Times in the Self-Paced Reading task by Korean L1/English L2 participants.

\begin{tabular}{lrrrr}
\hline & Sum Sq & Df & F value & $\operatorname{Pr}(>\mathrm{F})$ \\
\hline (Intercept) & 921382.81 & 1 & 8901314.55 & 0.0000 \\
logprevRT & 639.02 & 1 & 6173.45 & 0.0000 \\
ftp & 7.81 & 1 & 75.41 & 0.0000 \\
L2read & 67.69 & 1 & 653.90 & 0.0000 \\
nrchar & 41.90 & 1 & 404.79 & 0.0000 \\
SLstrength & 4.17 & 1 & 40.26 & 0.0000 \\
wordnum & 1.33 & 1 & 12.83 & 0.0003 \\
btp & 2.03 & 1 & 19.63 & 0.0000 \\
prevnrchar & 0.64 & 1 & 6.18 & 0.0129 \\
ftp:L2read & 3.82 & 1 & 36.91 & 0.0000 \\
SLstrength:wordnum & 1.66 & 1 & 16.04 & 0.0001 \\
ftp:btp & 1.66 & 1 & 16.05 & 0.0001 \\
L2read:btp & 0.88 & 1 & 8.50 & 0.0036 \\
L2read:wordnum & 0.20 & 1 & 1.97 & 0.1604 \\
ftp:wordnum & 0.15 & 1 & 1.42 & 0.2338 \\
ftp:SLstrength & 0.14 & 1 & 1.35 & 0.2458 \\
L2read:SLstrength & 0.03 & 1 & 0.32 & 0.5711 \\
SLstrength:btp & 0.03 & 1 & 0.26 & 0.6103 \\
wordnum:btp & 0.00 & 1 & 0.01 & 0.9083 \\
Residuals & 2684.24 & 25932 & & \\
\hline & & & &
\end{tabular}



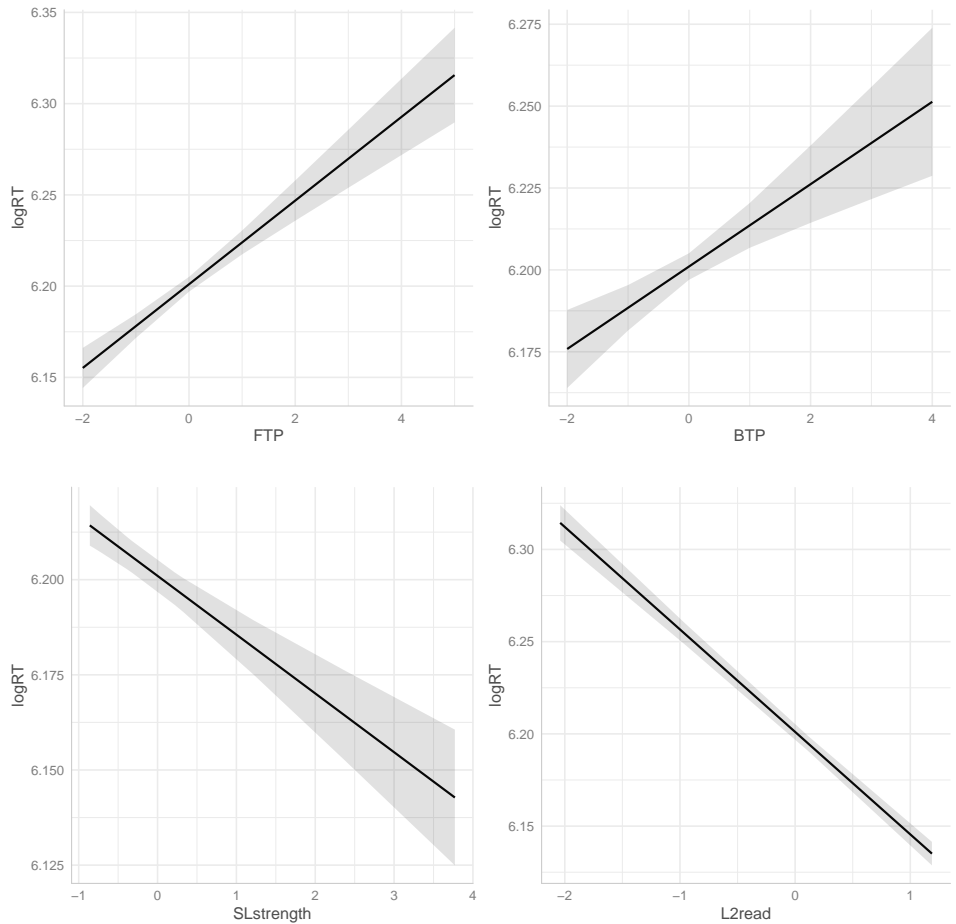

Figure 1: Marginal single term effects on reading times in the most parsimonious mixed-effect model 

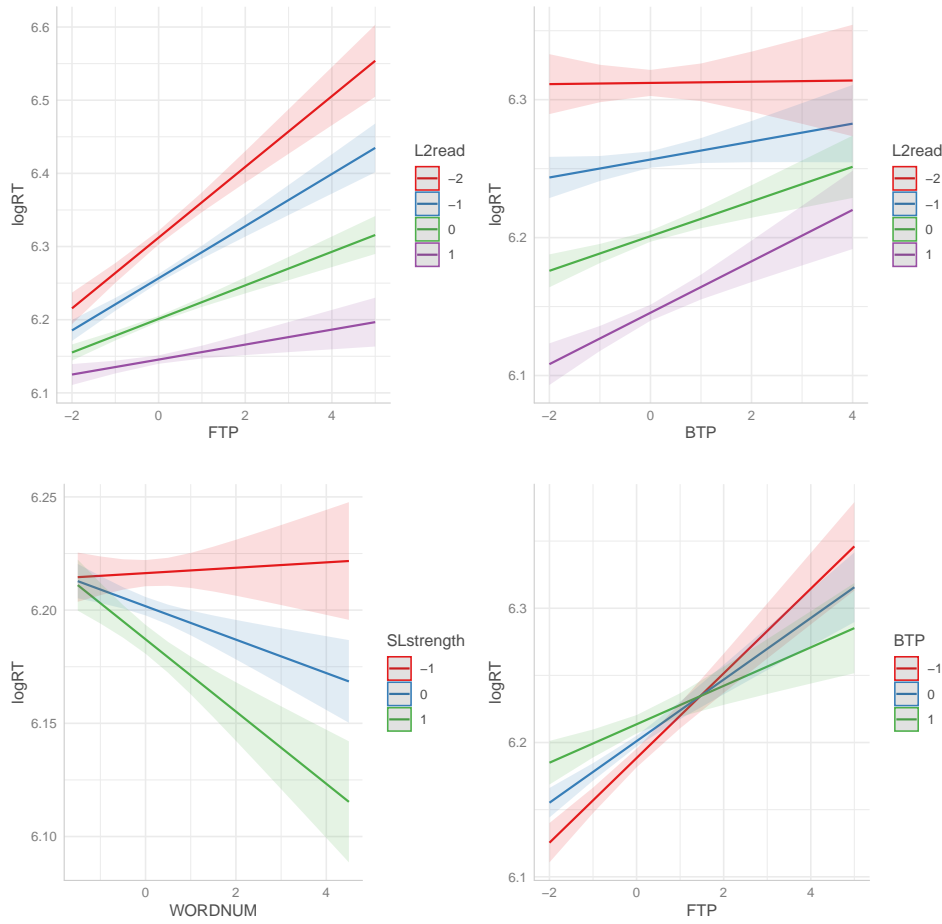

Figure 2: Marginal interaction effects on reading times in the most parsimonious mixed-effect model 


\section{Discussion}

What basic mechanisms support language learning and processing? As a higher-order cognitive function human language taps into a broad range of skills, some higher-order, some more basic - working under the hood of language at all times. The inherent fleeting nature of language in both the oral and written modalities requires sensitivity to sequentially relevant information, and thus sequential probabilistic constraints in language are likely to play a role in both acquisition and processing.

Accordingly, in this study we asked whether implicit statistical learning is associated with individual differences in second-language online reading. The rationale is that if SL subserves language learning and use, and learning languages implies the discovery of distributional relations, then greater individual sensitivity to distributional patterns in an artificial language task should be correlated with increased sensitivity to the lexical probabilities in a natural language, leading to greater reading efficiency.

In line with our hypothesis, second language readers with a highest score on an SL task read words faster in a self-paced reading task, as one would expect more efficient readers (i.e., those who make more optimal use of language statistics) to be faster readers. Furthermore, L2 readers were shown to be sensitive to immediate predictable word relations. In keeping with L1 research, we used the surprisal of a word as the negative logarithm of its conditional probability, that is the probability that the word will occur given the preceding context. Surprisal was calculated over a large corpus of English. Accordingly our L2 readers read faster words with lower surprisal. This sensitivity occurred for both forward and backward surprisal, suggesting that these proficient L2 readers had internalised fine-grained sensitivity of the directional relations between words.

Research in L1 sentence processing has shown that a word's probability/surprisal in context is strongly related to the amount of time it takes to read it. Our findings suggest that this may also be true of advanced L2 readers, who may read text similar to how L1 readers do. Further empirical work could compare read- 
ing patterns more directly between L1 and L2 speakers on the same sentences, something we did not do in our study. In addition, our measure of lexical predictability was limited to immediate local information, namely adjacent bigrams. An open question is whether L2 speakers are able to incorporate longer range statistics effectively, such as surprisal based on forward and backward probabilities of trigrams, fourgrams, and fivegrams. Future studies could build on the current one to test these hypotheses. Future studies could also elucidate the relation between SL and reading in L1 and L2 in the same individuals. A parsimonious theory of language would predict SL to be related to L2 reading via the mediating role of L1 reading. That is, SL abilities build L1 reading which in turn helps build L2 reading skills. These scenarios can be investigated in future studies in which bilingual individuals can be tested on SL tasks, and L1 and L2 reading tasks.

In the Introduction it was noted that predictability in L2 speakers has been empirically observed by some researchers, while it has been found inferior to native-like L1 abilities by others, and such inconsistent evidence leaves the debate unsettled. Several factors that were proposed to modulate predictive abilities in L2 are the amount of exposure to the language and the degree of proficiency of the learners (Kaan, 2014), learners' awareness to the relationship between the currently-given linguistic cue and upcoming information (Curcic et al., 2019), and language similarity between L1 and L2, with more similar languages being associated with predictability effects (Van Bergen \& Flecken, 2017). Our study suggests that predictability can occur in L2 processing even when L2 readers are not involved in conscious awareness processes (contrary to Curcic et al. 2019) and when L1 and L2 are rather dissimilar, as in the case of our Korean L1 / English L2 participants (contrary to Van Bergen \& Flecken 2017). Thus, it seems more plausible that sensitivity to predictability in L2 is mediated by degree of L2 experience, as our results also suggest. In that respect, our finding that the effect of forward conditional probability was stronger for less proficient readers, while the effect of backward conditional probability was stronger for more proficient L2 readers is intriguing in light of separate find- 
ings that backward probability is more informative in English, while forward probability is more informative in Korean (Onnis \& Thiessen, 2013; Thiessen et al. 2019), due to the specific word orders that each language exhibits. As a head-first language, English for example arranges prepositional phrases like "to school" such that the head "to" precedes the noun "school", while languages like Korean favor postpositional organization (as in hakkjo 'school' in - hakkjo-e 'school to'). Large-scale corpus analyses (Onnis \& Thiessen, 2013) show that English word clusters are more syntactically cohesive in a backward-going direction. For example, in a phrase like "to school," "to" does not strongly predict any word - because many nouns can follow "to" - but "school" more strongly retrodicts "to" because there is a relatively smaller set of words that can precede "school." The opposite obtains for Korean. Combining this finding with our findings in the current study, one possibility is that Korean L1 / English L2 speakers might develop a shifting sensitivity from the more Korean-like informative forward conditional probabilities, to the more informative backward conditional probabilities over time, requiring more experience. However, we note that our participants were all fairly proficient users of English, and thus future work should compare groups of L2 learners with more marked proficiency differences to establish shifting sensitivities to probabilistic information.

Is statistical learning a useful and valid construct to characterise a form of aptitude for second language outcomes? Certainly this study extends on recent literature relating SL and literacy development. Arciuli and Simpson (2012) found a correlation between performance in a non-linguistic SL task and measures of reading abilities derived from standardized reading tests in both elementary school children and adult native speakers. In addition, Spencer et al. (2015) established correlations between SL measures and early skills related to literacy development, notably oral language abilities, vocabulary knowledge, and phonological processing. With respect to second language learning, Frost et al. (2013) provided evidence that SL abilities predict single word decoding abilities in a second language. These studies established relations between SL performance and either broad measures of literacy outcomes, such as scores of 
standardized reading tests, or measures of single-word orthographic knowledge. With respect to previous studies the present one allowed a finer-grained examination of the role of statistical learning in more naturalistic conditions of whole sentence online reading. Future studies could also investigate whether SL is an aptitude distinct and distinguishable from Working Memory, which robustly predicts L2 outcomes (Linck et al., 2014).

Methodologically, our study helped obviate one major drawback deriving from tasks typically employed in expectation-based experiments: the constraining and often artificial nature of the linguistic stimuli. Huettig \& Mani (2016) for example, pointed out that visual stimuli presented in visual-world eye-tracking experiments on prediction may provide critical scaffolding for the finding of such effects, because the words showing quicker eye-gazing fixations are preceded by pictures of the objects those words refer to. Other studies of reading focus on manipulating artificially constructed minimal-pair sentences that contain one of two words, one more predictable than the other on the basis of cloze probability Huettig \& Mani (2016). Similarly, Brothers et al. (2017) provide evidence that the task influences the extent to which readers engage in lexical prediction. Thus, the evidence for expectation-based language processing is often limited to circumscribed stimuli. Our study obviates to such limited and limiting circumstances by predicting general reading patterns not just on specific target words, but over entire sentences sampled from authentic written sources. The effects of forward and backward surprisal on reading times thus appear more robust and generalizable.

\subsection{Conclusion}

We have argued that the ability to track statistical relations in sequenced patterns may not only be useful in learning linguistic structure, but is also significantly correlated with the ability to process natural language as adults. Finding correlations between artificial grammar learning proclivities and language processing abilities contributes to validating the statistical learning approach to language. Future work could help establish whether people who are more sen- 
sitive to statistical sequential information make better language learners, and ultimately language users. In addition, understanding exactly what type of statistical information is required to optimize language tasks such as reading would greatly expand our knowledge of the mechanisms required for language processing. This line of research is not only useful to inform theories of language in the brain, but has potential practical applications. For example, it may be possible to assist inefficient second-language readers by helping them process statistical information more optimally.

\section{References}

Abla, D., Katahira, K., \& Okanoya, K. (2008). On-line assessment of statistical learning by event-related potentials. Journal of Cognitive Neuroscience, 20, 952-964.

Arciuli, J., \& Simpson, I. C. (2012). Statistical learning is related to reading ability in children and adults. Cognitive science, 36, 286-304.

Baayen, R. H., \& Milin, P. (2010). Analyzing reaction times. International Journal of Psychological Research, 3, 12-28.

Bates, D., Maechler, M., Bolker, B., Walker, S., Christensen, R. H. B., Singmann, H., Dai, B., Grothendieck, G., Green, P., \& Bolker, M. B. (2015). Package 'Ime4'. Convergence, 12, 2.

Brothers, T., Swaab, T. Y., \& Traxler, M. J. (2017). Goals and strategies influence lexical prediction during sentence comprehension. Journal of Memory and Language, 93, 203-216.

Brysbaert, M. (2019). How many words do we read per minute? a review and meta-analysis of reading rate. Journal of Memory and Language, 109, 104047. 
Christiansen, M. H., Conway, C. M., \& Onnis, L. (2012). Similar neural correlates for language and sequential learning: Evidence from event-related brain potentials. Language and cognitive processes, 27, 231-256.

Curcic, M., Andringa, S., \& Kuiken, F. (2019). The role of awareness and cognitive aptitudes in 12 predictive language processing. Language Learning, $69,42-71$.

Dussias, P. E., Kroff, J. R. V., Tamargo, R. E. G., \& Gerfen, C. (2013). When gender and looking go hand in hand: Grammatical gender processing in 12 spanish. Studies in Second Language Acquisition, 35, 353-387.

Dutoit, T., Pagel, V., Pierret, N., Bataille, F., \& Van der Vrecken, O. (1996). The mbrola project: Towards a set of high quality speech synthesizers free of use for non commercial purposes. In Spoken Language, 1996. ICSLP 96. Proceedings., Fourth International Conference on (pp. 1393-1396). IEEE volume 3 .

Erdos, C., Genesee, F., Savage, R., \& Haigh, C. A. (2011). Individual differences in second language reading outcomes. International Journal of Bilingualism, $15,3-25$.

Evans, J. L., Saffran, J. R., \& Robe-Torres, K. (2009). Statistical learning in children with specific language impairment. Journal of Speech, Language, and Hearing Research, .

Fine, A. B., Jaeger, T. F., Farmer, T. A., \& Qian, T. (2013). Rapid expectation adaptation during syntactic comprehension. PloS one, 8 .

Frank, S. L. (2013). Uncertainty reduction as a measure of cognitive load in sentence comprehension. Topics in Cognitive Science, 5, 475-494.

Frank, S. L., Monsalve, I. F., Thompson, R. L., \& Vigliocco, G. (2013). Reading time data for evaluating broad-coverage models of english sentence processing. Behavior Research Methods, 45, 1182-1190. 
Fraundorf, S. H., \& Jaeger, T. F. (2016). Readers generalize adaptation to newly-encountered dialectal structures to other unfamiliar structures. Journal of Memory and Language, 91, 28-58.

Frost, R., Siegelman, N., Narkiss, A., \& Afek, L. (2013). What predicts successful literacy acquisition in a second language? Psychological science, 24, $1243-1252$.

Gómez, R. L., \& Gerken, L. (2000). Infant artificial language learning and language acquisition. Trends in cognitive sciences, 4, 178-186.

Goodkind, A., \& Bicknell, K. (2018). Predictive power of word surprisal for reading times is a linear function of language model quality. In Proceedings of the 8th workshop on cognitive modeling and computational linguistics (CMCL 2018) (pp. 10-18).

Grüter, T., Lew-Williams, C., \& Fernald, A. (2012). Grammatical gender in 12: A production or a real-time processing problem? Second Language Research, 28, 191-215.

Grüter, T., \& Rohde, H. (2013). L2 processing is affected by rage: Evidence from reference resolution. In the 12th conference on Generative Approaches to Second Language Acquisition (GASLA).

Hopp, H. (2013). Grammatical gender in adult 12 acquisition: Relations between lexical and syntactic variability. Second Language Research, 29, 33-56.

Huettig, F., \& Mani, N. (2016). Is prediction necessary to understand language? probably not. Language, Cognition and Neuroscience, 31, 19-31.

Ito, A., Corley, M., \& Pickering, M. J. (2018). A cognitive load delays predictive eye movements similarly during 11 and 12 comprehension. Bilingualism: Language and Cognition, 21, 251-264.

Jones, J., \& Pashler, H. (2007). Is the mind inherently forward looking? comparing prediction and retrodiction. Psychonomic bulletin \& review, 14, 295-300. 
Jurafsky, D., Bell, A., Gregory, M., \& Raymond, W. D. (2001). Probabilistic relations between words: Evidence from reduction in lexical production. Typological studies in language, 45, 229-254.

Kaan, E. (2014). Predictive sentence processing in 12 and 11: What is different? Linguistic Approaches to Bilingualism, 4, 257-282.

Kaufman, S. B., DeYoung, C. G., Gray, J. R., Jiménez, L., Brown, J., \& Mackintosh, N. (2010). Implicit learning as an ability. Cognition, 116, 321-340.

Leal, T., Slabakova, R., \& Farmer, T. A. (2017). The fine-tuning of linguistic expectations over the course of 12 learning. Studies in Second Language Acquisition, 39, 493-525.

Levy, R. (2008). Expectation-based syntactic comprehension. Cognition, 106, $1126-1177$.

Lew-Williams, C., \& Fernald, A. (2010). Real-time processing of gender-marked articles by native and non-native spanish speakers. Journal of memory and language, 63, 447-464.

Linck, J. A., Osthus, P., Koeth, J. T., \& Bunting, M. F. (2014). Working memory and second language comprehension and production: A meta-analysis. Psychonomic bulletin \&5 review, 21, 861-883.

Marslen-Wilson, W. (1973). Linguistic structure and speech shadowing at very short latencies. Nature, 244, 522-523.

Martin, C. D., Thierry, G., Kuipers, J.-R., Boutonnet, B., Foucart, A., \& Costa, A. (2013). Bilinguals reading in their second language do not predict upcoming words as native readers do. Journal of Memory and Language, 69, $574-588$.

McDonald, S. A., \& Shillcock, R. C. (2003a). Eye movements reveal the on-line computation of lexical probabilities during reading. Psychological science, 14, 648-652. 
McDonald, S. A., \& Shillcock, R. C. (2003b). Low-level predictive inference in reading: The influence of transitional probabilities on eye movements. Vision Research, 43, 1735-1751.

Mitsugi, S., \& Macwhinney, B. (2016). The use of case marking for predictive processing in second language japanese. Bilingualism: Language and Cognition, 19, 19-35.

Moers, C., Meyer, A., \& Janse, E. (2017). Effects of word frequency and transitional probability on word reading durations of younger and older speakers. Language and speech, 60, 289-317.

Nakazawa, M. (2007). Practices of medical and health data analysis using r.

Onnis, L., Cheung, S., \& Huettig, F. (2020). More than memory: Key evidence for multi-word storage may be effects of prediction and postdiction in disguise. Submitted manuscript, .

Onnis, L., \& Huettig, F. (2020). Is language processing inherently forward looking? Submitted manuscript, .

Onnis, L., \& Thiessen, E. (2013). Language experience changes subsequent learning. Cognition, 126, 268-284.

Pelucchi, B., Hay, J. F., \& Saffran, J. R. (2009). Learning in reverse: Eightmonth-old infants track backward transitional probabilities. Cognition, 113, $244-247$.

Perruchet, P., \& Desaulty, S. (2008). A role for backward transitional probabilities in word segmentation? Memory $\mathscr{E}$ cognition, 36, 1299-1305.

Pickering, M. J., \& Gambi, C. (2018). Predicting while comprehending language: A theory and review. Psychological Bulletin, 144, 1002.

Smith, N. J., \& Levy, R. (2013). The effect of word predictability on reading time is logarithmic. Cognition, 128, 302-319. 
Spencer, M., Kaschak, M. P., Jones, J. L., \& Lonigan, C. J. (2015). Statistical learning is related to early literacy-related skills. Reading and writing, 28, $467-490$.

Staub, A. (2015). The effect of lexical predictability on eye movements in reading: Critical review and theoretical interpretation. Language and Linguistics Compass, 9, 311-327.

Thiessen, E. D., Onnis, L., Hong, S.-J., \& Lee, K.-S. (2019). Early developing syntactic knowledge influences sequential statistical learning in infancy. Journal of experimental child psychology, 177, 211-221.

Van Bergen, G., \& Flecken, M. (2017). Putting things in new places: Linguistic experience modulates the predictive power of placement verb semantics. Journal of Memory and Language, 92, 26-42.

Voeten, C. (2019). buildmer: Stepwise elimination and term reordering for mixed-effects regression. $R$ package version, 1 .

Willems, R. M., Frank, S. L., Nijhof, A. D., Hagoort, P., \& Van den Bosch, A. (2016). Prediction during natural language comprehension. Cerebral Cortex, 26, 2506-2516.

Yim, D., \& Windsor, J. (2010). The roles of nonlinguistic statistical learning and memory in language skill. Communication Sciences 83 Disorders, 15, 381-396. 